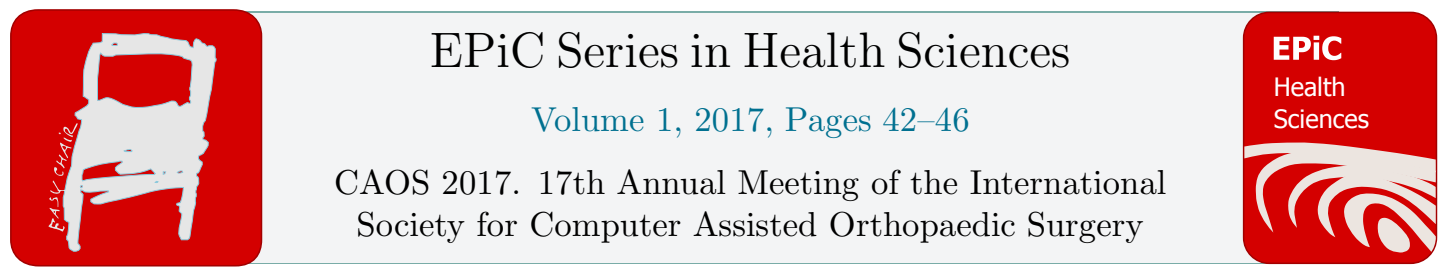

\title{
Atlas-based Reconstruction of 3D Intensity Volumes of Lower Extremity from 2D Calibrated X-ray Images
}

\author{
Weimin $\mathrm{Yu}^{1}$ and Guoyan Zheng ${ }^{1}$ \\ Institute for Surgical Technology and Biomechanics, University of Bern, Bern, Switzerland \\ weimin.yu@istb.unibe.ch \\ guoyan.zheng@istb.unibe.ch
}

\begin{abstract}
A new atlas-based 2D-3D reconstruction of 3D volumes of lower extremity from a pair of calibrated X-ray images was presented. The approach combines non-rigid 2D$2 \mathrm{D}$ registration based 3D landmark reconstruction with the B-spline parametrization of TPS transformation, incorporating the smoothness property of B-splines for regularization. Efficacy of the present approach was evaluated on the calibrated X-ray images and CT data. Also, we take the knee joint articulation into consideration. Articulated B-spline parameterization leads to the almost same accuracy as individual B-spline parameterization and has the superiority over the latter when it comes to the prevention from the knee joint penetration.
\end{abstract}

\section{Introduction}

Accurate 3D pre-operative planning shows significance of improving the precision of Total Hip Arthroplasty (THA) and Total Knee Arthroplasty (TKA). Since CT acquisition leads to high radiation exposure to patients, it is clinically desirable to find an alternative to CT scan for planning THA or TKA such as patient-specific 2D-3D reconstruction from a limited number of 2D calibrated X-ray images acquired with much lower radiation dose e.g. EOS imaging [2]. Feature-based 2D-3D non-rigid registration based on the construction of statistical shape model (SSM) as a priori has been applied to reconstruct the surface models of proximal femur [4], and also the surface reconstruction of lower extremity for TKA has been validated in a cadaveric study [3]. On the other hand, intensity-based 2D-3D non-rigid registration can reconstruct the patient-specific intensity volumes like CT data to have an insight into 3D intensity distribution and morphology such as intramedullary anatomy, which can provide more comprehensive information in routine clinical practice. In this study, we will present an atlas-based 2D-3D reconstruction method and introduce its application to reconstruct the intensity volumes of lower extremity. Moreover, we take the articulation in the knee joint into consideration so as to avoid the penetration between femur and tibia which is favorable for the pre-operative planning. 


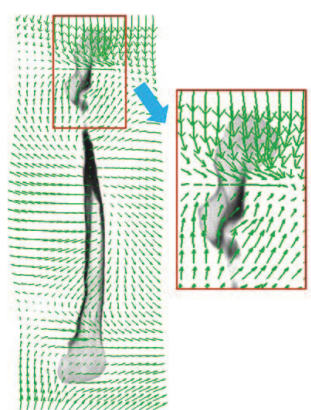

(a) Voxel-wise $3 \mathrm{D}$ displacement field computed from the TPS tranformation

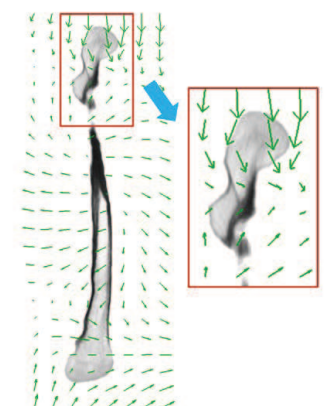

(b) Displacements of the B-spline control points interpolated from the TPS tranformation
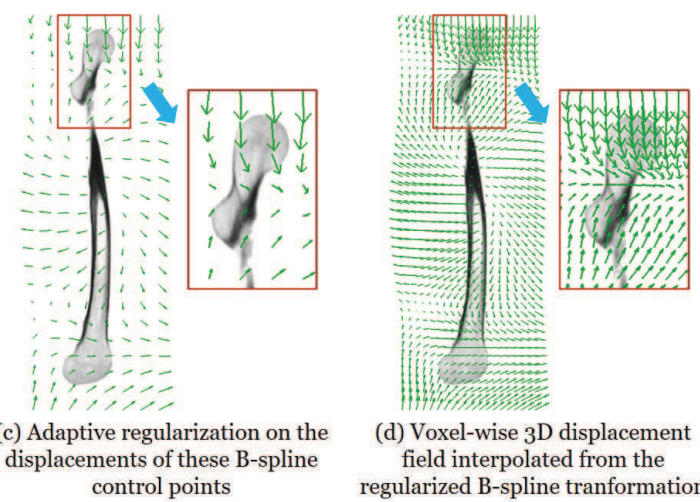

(d) Voxel-wise $3 \mathrm{D}$ displacement field interpolated from the regularized B-spline tranformation

Figure 1: A comparison of the 3D deformation fields derived from interpolation of a TPS transformation and from the B-spline parameterization of a TPS transformation

\section{Materials and Methods}

The proposed method takes two calibrated X-ray images as the input and deformably fits an intensity volume atlas including a femur template and a tibia template (both templates maintain the relative positions) to the given images. For each template, a set of landmarks was extracted from the outer surface as well as the intramedullary canal surface, and then the atlas-based 2D-3D reconstruction method can be summarized with 4 steps: 1) 2D-2D non-rigid registration between the given X-ray images and the simulated radiographic images generated from either the femur template or the tibia template; 2) the extracted landmarks are projected onto the image planes and are transformed with the obtained 2D deformation fields in step 1, followed by a triangulation-based reconstruction process for each landmark; 3) two individual thin-platespline (TPS) transformations (femur \& tibia) can be computed from the original landmark sets and the newly reconstructed landmark sets; 4) a B-spline parameterization process is done to fit a regularized B-spline transformation from the TPS transformations.

Since 2D-2D non-rigid registration is prone to find incorrect correspondences when the Xray images suffer from noise, artifact and low contrast, which could lead to an inaccurate TPS transformation. Thus, the B-spline parameterization strategy is adapted for regularization due to the smoothness property of B-splines. Furthermore, an adaptive regularization [1] is introduced for a better behavior of the displacement vectors on the B-spline grid (see Figure 1). In order to avoid the potential penetration between femur and tibia, an articulated B-spline parameterization strategy takes both TPS transformations into account with a pre-defined splitting plane from the femur \& tibia templates. When the B-spline control points are above the plane, the displacements are only determined by the TPS transformation of femur, otherwise, these points are only determined by the TPS transformation of tibia.

\section{Results}

10 cadaveric $\mathrm{CT}$ data of lower extremity were collected for evaluating the proposed method. For each case, two simulated radiographic images were generated from the associated CT volume as the input, and the binary labels of femur and tibia as well as the cortical bone regions were manually segmented from the associated CT volume as the ground truth. Average surface dis- 
tance (ASD) and dice coefficient (DC) were used to evaluate the overall volume reconstruction accuracy of femur and tibia, and to assess the cortical bone region reconstruction accuracy, cortical bone region ASD (CBRASD) and cortical bone region DC (CBRDC) were also measured. We compared the results computed from the articulated B-spline parameterization strategy with the results obtained from the individual B-spline parameterization strategy. According to Table 1, articulated B-spline parameterization leads to the almost same accuracy as individual B-spline parameterization and has the superiority over the latter when it comes to the prevention from the knee joint penetration (see Figure 2).

Table 1: Quantitative results of the experiment conducted on 10 cadaveric legs.

\begin{tabular}{|c|c|c|c|c|c|c|c|c|c|c|c|}
\hline \multicolumn{12}{|c|}{ Individual B-spline Parameterization } \\
\hline \multicolumn{6}{|c|}{ Femur } & \multicolumn{6}{|c|}{ Tibia } \\
\hline & $\begin{array}{l}\text { ASD } \\
(\mathrm{mm})\end{array}$ & $\begin{array}{l}\text { DC } \\
(\%)\end{array}$ & $\begin{array}{c}\text { I-CBRASD } \\
(\mathrm{mm})\end{array}$ & $\begin{array}{c}\text { CBRASD } \\
(\mathrm{mm})\end{array}$ & $\begin{array}{c}\text { CBRDC } \\
(\%)\end{array}$ & & $\begin{array}{l}\text { ASD } \\
(\mathrm{mm})\end{array}$ & $\begin{array}{l}\mathrm{DC} \\
(\%)\end{array}$ & $\begin{array}{c}\text { I-CBRASD } \\
(\mathrm{mm})\end{array}$ & \begin{tabular}{|c|} 
CBRASD \\
$(\mathrm{mm})$
\end{tabular} & $\begin{array}{c}\text { CBRDC } \\
(\%)\end{array}$ \\
\hline Mean & 1.5 & 91.9 & 0.8 & 1.0 & 84.3 & Mean & 1.3 & 91.6 & 1.1 & 1.0 & 79.1 \\
\hline Std & 0.2 & 0.8 & 0.2 & 0.2 & 2.7 & Std & 0.2 & 1.1 & 0.3 & 0.2 & 3.4 \\
\hline \multicolumn{12}{|c|}{ Articulated B-spline Parameterization } \\
\hline \multicolumn{6}{|c|}{ Femur } & \multicolumn{6}{|c|}{ Tibia } \\
\hline & $\begin{array}{l}\text { ASD } \\
(\mathrm{mm})\end{array}$ & $\begin{array}{l}\text { DC } \\
(\%)\end{array}$ & $\begin{array}{c}\text { I-CBRASD } \\
(\mathrm{mm})\end{array}$ & \begin{tabular}{|c|} 
CBRASD \\
$(\mathrm{mm})$
\end{tabular} & $\begin{array}{c}\text { CBRDC } \\
(\%)\end{array}$ & & $\begin{array}{l}\text { ASD } \\
(\mathrm{mm})\end{array}$ & $\begin{array}{l}\mathrm{DC} \\
(\%)\end{array}$ & $\begin{array}{c}\text { I-CBRASD } \\
(\mathrm{mm})\end{array}$ & \begin{tabular}{|c|} 
CBRASD \\
$(\mathrm{mm})$
\end{tabular} & $\begin{array}{c}\text { CBRDC } \\
(\%)\end{array}$ \\
\hline Mean & 1.5 & 91.8 & 0.7 & 1.0 & 84.3 & Mean & 1.3 & 91.4 & 1.1 & 1.0 & 79.0 \\
\hline Std & 0.2 & 0.8 & 0.2 & 0.2 & 2.7 & Std & 0.2 & 1.1 & 0.3 & 0.2 & 3.4 \\
\hline
\end{tabular}

\section{Discussions}

An atlas-based approach for reconstructing 3D volumes of a complete lower extremity from a pair of calibrated X-ray images was presented in the abstract. In comparison to our previously developed B-spline control point based 2D-3D reconstruction method [5] where the uniform distribution of the $3 \mathrm{D}$ control points are assigned, the probability of the $2 \mathrm{D}$ projections of these points onto high gradient regions is relatively low, leading to an inefficient force to update the projected locations, while in this proposed method, all 3D landmarks from outer surfaces and intramedullary canal surfaces are extracted to drive the 2D-3D registration, which may lead to a better performance. In addition, an articulated B-spline parameterization strategy is adapted to avoid the penetration of femur and tibia which is not considered in our previous work [5], and the results of the experiments demonstrated the efficacy of the proposed method.

\section{References}

[1] Myronenko A and Song X. Adaptive regularization of ill-posed problems: application to non-rigid image registration. arXiv:0906.3323 https://arxiv.org/abs/0906.3323, 2009.

[2] McKenna C, Wade R, Faria R, Yang H, Stirk L, Gummerson N, Sculpher M, and Woolacott N. Eos 2d/3d x-ray imaging system: A systematic review and economic evaluation. Health Technology Assessment 16(14), https://www.ncbi.nlm.nih.gov/books/NBK97733/, 2012.

[3] Zheng G, Nolte LP, and Jaramaz B. Patient-specific 3d reconstruction of a complete lower extremity from 2d x-rays: A cadaveric validation study. The Bone \& Joint Journal 98(9), http://www.bjjprocs.boneandjoint.org.uk/content/98-B/SUPP_5/30, 2016. 


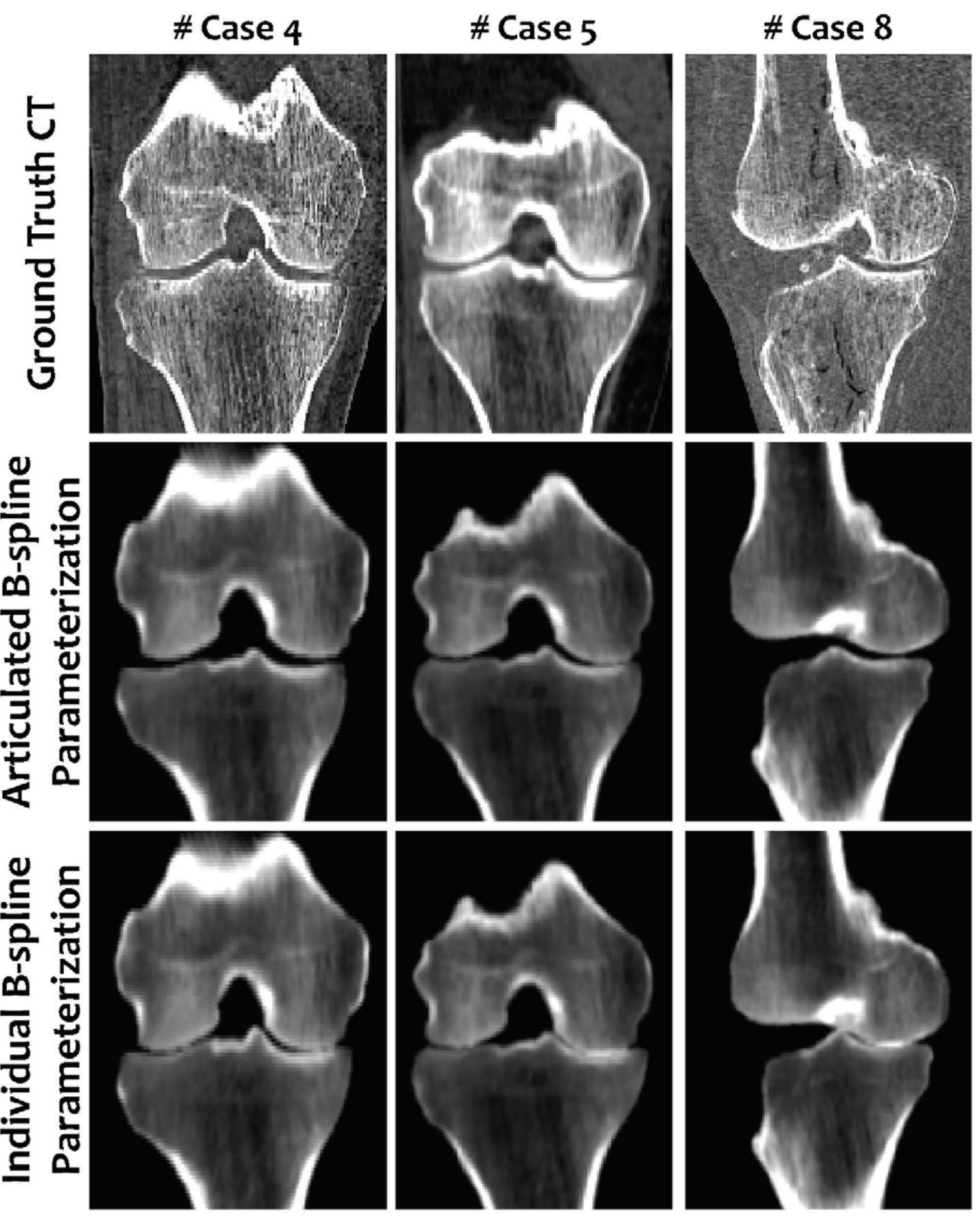

Figure 2: A qualitative comparison of the knee joint reconstruction using the individual \& articulated B-spline parameterization strategies. 
[4] Zheng G, Gollmer S, Schumann S, Dong X, Feilkas T, , and Gonzlez Ballester M. A 2d/3d correspondence building method for reconstruction of a patient-specific $3 \mathrm{~d}$ bone surface model using point distribution models and calibrated x-ray images. Medical Image Analysis 13(6), http://www.sciencedirect.com/science/article/pii/S1361841508001448, 2009.

[5] Yu W, Chu C, Tannast M, and Zheng G. Fully automatic reconstruction of personalized 3d volumes of the proximal femur from $2 \mathrm{~d}$ x-ray images. International Journal of Computer Assisted Radiology and Surgery 11(9), https://link.springer.com/article/10.1007/s11548-016-1400-9, 2016. 